\title{
Hepatic resection for isolated stomach cancer liver metastases: A single-center experience
}

Hyunyou Kim, Hyung-II Seo

Department of Surgery, Biomedical Research Institute, Pusan National University Hospital, Busan, Korea

Purpose: The objective of this study was to investigate the outcomes of selected patients with stomach cancer liver metastasis (SCLM) without extrahepatic metastases after hepatic resection.

Methods: Patients whose imaging results did not detect extrahepatic disease were selected for hepatic resection. If R0 resection was possible and if the operative risk was low in the preoperative tests, the patients underwent hepatic resection.

Results: Between 2011 and 2016, seven patients underwent hepatic resection for SCLM. All patients received hepatic resection to achieve an RO resection. Minor liver resection was performed in all patients. Two patients showed long-term survival with a single lesion and human epidermal growth factor receptor 2 (HER2)-negative tumor. The 5-year overall survival and disease-free survival rates after hepatic resection were $38.1 \%$ and $28.6 \%$, respectively.

Conclusion: Hepatic resection for isolated SCLM may be considered as a multimodality treatment. However, it has only limited benefits in select patients. It has long-term survival benefit in patients with single metastases and HER2-negative hormonal status.

Keywords: Liver resection, Liver metastases

\section{INTRODUCTION}

Stomach cancer is the second most common cause of cancer related death worldwide. The liver is the major site of metastases in $9 \%-40 \%$ of cases, though the metastatic pattern in most cases is diffuse, involving both the peritoneum and distant lymph nodes [1-4].

In contrast to the well-described guidelines for hepatic resection of colorectal cancer and neuroendocrine liver metastases, surgical resection of stomach cancer liver metastases (SCLMs) is only considered in a select few patients. Treatment for SCLM is mainly based on the physician's experience because of the limited clinical benefit of hepatic resection. In general, surgical resection is not

Received: Jun 28, 2019 Revised: Dec 12, 2019 Accepted: Dec 17, 2019 Correspondence to: Hyung-II Seo

Department of Surgery, Biomedical Research Institute, Pusan National

University Hospital, 179 Gudeok-ro, Seo-gu, Busan 49241, Korea

Tel: +82-51-240-7238, Fax: +82-51-247-1365

E-mail: seohi71@hanmail.net

ORCID: Hyunyou Kim (https://orcid.org/0000-0002-1656-2337), Hyung-II Seo (https://orcid.org/0000-0002-4132-7662)

Copyright @ 2019 Korean Society of Surgical Oncology

This is an Open Access article distributed under the terms of the Creative Commons Attribution Non-Commercial License (http://creativecommons.org/licenses/by-nc/4.0) which permits unrestricted non-commercial use, distribution, and reproduction in any medium, provided the original work is properly cited. commonly performed in patients with hepatic metastases from gastric cancers, because of poor long-term outcomes. However, recent studies suggest that hepatic resection for SCLM showed improved outcomes in select patients, with a 5-year survival ranging from $23 \%$ to $42 \%$ [3-6]. Yet, the effectiveness of liver resection in the treatment of SCLM is still controversial. The aim of this paper was to review the outcomes of select patients with SCLM after hepatic resection at a single center.

\section{METHODS}

\section{Patients}

Seven patients with solitary hepatic metastasis for SCLM (excluding liver directed invasion) underwent hepatic resection between 2011 and January 2016. Patients were selected for hepatic resection by evaluating them for the possibility of performing R0 resection in serological assessment and imaging studies, such as computed tomography (CT), magnetic resonance imaging, bone scintigraphy, and positron emission tomography-CT (PET/CT). If it was possible to perform $\mathrm{R} 0$ resection and there was a low operative risk according to the American Society of Anesthesiologists physical status I or II during preoperative tests, the patient underwent surgery. This retrospective study was approved by the Institutional Review Board at Pusan National University Hospital Clinical Trial 
Center (IRB No. H- 2001-001-086), and written informed consent was obtained from all study participants.

\section{Postoperative follow-up schedule}

Patients underwent a follow-up examination every 6 months, which included the following examinations: physical and laboratory testing (serology, liver function, and tumor marker expression) and radiographic examinations (chest X-ray, CT, and PET/CT).

\section{Statistical analysis}

Overall survival (OS) and disease-free survival (DFS) rates were analyzed using Kaplan-Meier charts, and the statistical significance was determined by a log-rank test, with a two-tailed P-value $<0.05$ considered statistically significant. The statistical analysis was performed using SPSS statistical software version 12.0 (SPSS Inc., Chicago, IL, USA).

\section{RESULTS}

Seven patients underwent hepatic resection for SCLM between 2011 and January 2016. In one case (case 6) the stomach cancer staging and biologic data were unknown. Six patients' human epidermal growth factor receptor 2 (HER2) status was evaluated, three patients were HER2 3+; this result was confirmed via biopsy specimen taken during liver resection. Synchronous disease was identified in two patients and metachronous disease was identified in five patients. Three patients diagnosed with metachronous disease received adjuvant chemotherapy after the gastrectomy (Table 1).

The median time interval between the initial diagnosis of stomach cancer and detection of hepatic metastases, excluding the two patients with synchronous disease, was 23 months. The physical status of the patients was not severe but mild to moderate according to the American Society of Anesthesiologists score. Minor resection (lesser than three segments) were performed in all patients, of which the median largest size was $2.3 \mathrm{~cm}$ (range, $0.6-4.5 \mathrm{~cm}$ ). The number of sites in the liver containing tumor metastases was from 1 to more than 10 . Four patients contained only 1 site of metastasis and case 4 had more than 10. Despite strict patient selection, three patients had multiple sites of liver disease (case 4, 5, and 7). Case 4 had an extrahepatic lesion found in the 2 nd operation. Six patients, except one patient who refused treatment, received

Table 1. Clinicopathological characteristics of primary disease

\begin{tabular}{llclccc}
\hline Case no. & Sex & Age $(\mathrm{yr})$ & TNM & HER2 & Adjuvant treatment & DFS (mo) \\
\hline 1 & Female & 66 & T3N3M1 & 0 & - & 0 \\
2 & Female & 61 & T4aNOM0 & 0 & XP & 26 \\
3 & Male & 65 & T2N1M0 & $3+$ & UF & 18 \\
4 & Male & 70 & T1aNOM0 & 0 & $3+$ & - \\
5 & Male & 73 & T4aN3aM1 & NA & - & 0 \\
6 & Male & 59 & NA & $3+$ & TS-1 & 11 \\
7 & Male & 59 & T3N2M0 & & 14 \\
\hline
\end{tabular}

TNM, tumor node metastasis according to American Joint Committee on Cancer (AJCC) 7th edition; HER2, human epidermal growth factor receptor 2; DFS, disease-free interval; NA, not available; XP, capecitabine+cisplatin; UFT, tegafur/uracil; TS-1, tegafur/gimeracil/oteracil K.

Table 2. Clinicopathological characteristics of the secondary disease

\begin{tabular}{|c|c|c|c|c|c|c|}
\hline Case no. & Type of second operation & Size $(\mathrm{cm})$ & No. of site & Adjuvant treatment & Recurrence ${ }^{a)}$ & Survival ${ }^{\text {a) }}$ \\
\hline 1 & Segmentectomy & 3.2 & 1 & CCRT (FP) & NED & Alive (78) \\
\hline 2 & Segmentectomy & 0.6 & 1 & FP & NED & Alive (60) \\
\hline 3 & LLS & 3.0 & 1 & CCRT & LN (6) & Dead (37) \\
\hline 4 & $\begin{array}{l}\text { LLS } \\
\text { Completion total gastrectomy } \\
\text { Right hemicolectomy }\end{array}$ & 2.0 & $>10$ & $\begin{array}{l}\text { CCRT (FP) } \\
\text { TS-1 } \\
\text { OLF }\end{array}$ & $\mathrm{CP}(7)$ & Dead (15) \\
\hline 5 & LLS & 1.5 & 3 & TS-1 & $\mathrm{CP}(1)$ & Dead (2) \\
\hline 6 & LLS & 4.5 & 1 & - & $\mathrm{CP}(10)$ & Dead (13) \\
\hline 7 & Segmentectomy & 1.5 & 2 & Herceptin+FP & Liver (21) & Alive (29) \\
\hline
\end{tabular}

Size, the largest size; CCRT, concurrent chemoradiation therapy; FP, 5-fluorouracil+cisplastin; NED, no evidence of disease; LLS, left lateral sectionectomy; LN, lymph node; $\mathrm{CP}$, carcinomatosis peritonei; TS-1, tegafur/gimeracil/oteracil K; OLF, oxaliplatin+5-fluorouracil; Herceptin, trastuzumab.

${ }^{a}$ The numbers in parentheses mean the months of recurrence and survival. 

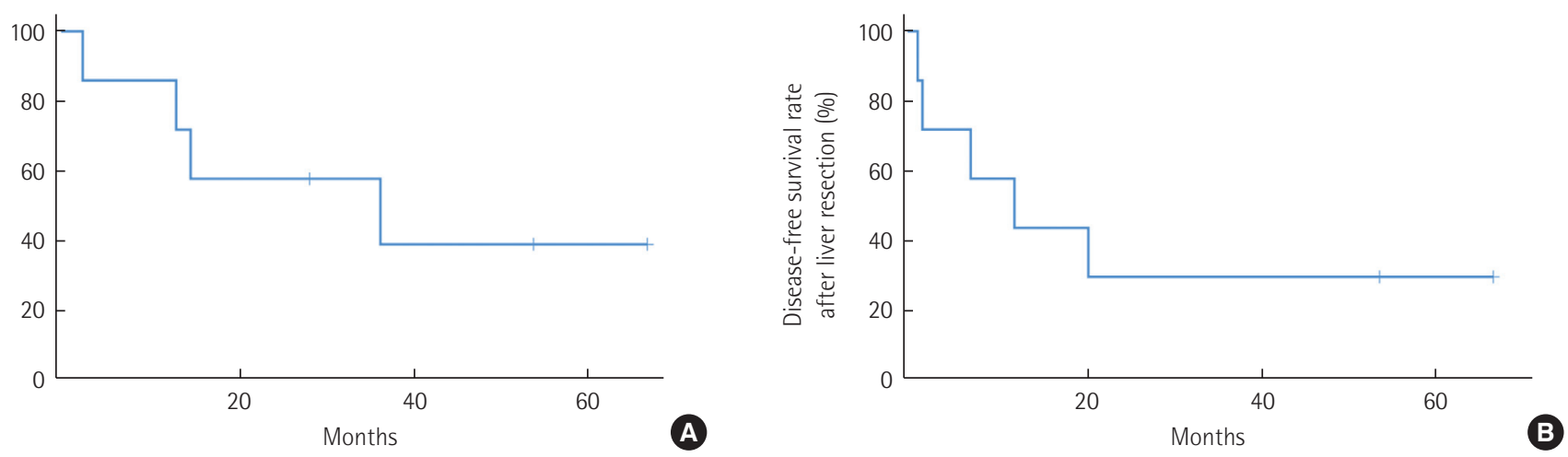

Fig. 1. (A) The 1-, 3-, and 5-year overall survival were $85.7 \%, 42.9 \%$, and $38.1 \%$, respectively. (B) The disease-free survival rates after hepatic resection were $28.6 \%$, respectively.

postoperative adjuvant chemotherapy following hepatic resection. Three patients received concurrent chemoradiation therapy (case 1,3 , and 4 ) and another three patients received only chemotherapy (case 2, 5, and 7); of these patients, case 3, 5, and 7 were HER2 3+. Trastuzumab was administrated after hepatic resection in only case 7. Case 4 and 5, who received adjuvant treatment after resection, were diagnosed with perineural invasion after analyses of resected liver specimen, and these patients showed an earlier and more aggressive pattern of recurrence. Case 6 had received only hepatic resection without any follow-up adjuvant treatment. Longterm survival was seen in single lesion and HER2-negative patients (case 1 and 2). The median survival was 33 months for resected patients (Table 2).

The OS after 1 year, 3 years, and 5 years were $85.7 \%, 42.9 \%$, and $38.1 \%$, respectively. The DFS rate after hepatic resection was $28.6 \%$ (Fig. 1).

\section{DISCUSSION}

In contrast to the well-described guidelines for hepatic resection of colorectal cancer and neuroendocrine cancer liver metastasis, hepatic resection of SCLM is mainly based on the experience of the institution and physician. Overall 5-year survival in patients with liver metastases ranged from $0 \%$ to $10 \%$ and hepatic resection was considered to not be beneficial in these patients. However, there are retrospective, non-randomized studies that reported an improvement in the survival of patients who underwent liver resection for SCLM. Two meta-analyses reported a 5-year survival of $23.8 \%-27.8 \%$ and a median survival of 22 to 29 months [3-5]. A Japanese group reported on their experience using an aggressive approach to treat SCLM, adopting the same patient selection criteria as used for colorectal liver metastases. The median recurrence-free survival was similar between both groups (15.2 months vs. 16.4 months), while the 5-year survival in SCLM patients was worse (55\% vs. 14\%) [4]. Our study satisfied this condition as the 5-year OS and DFS rates after hepatic resection were $38.1 \%$ and $28.6 \%$. Unfortunately, there is limited data on liver resection in SCLM because there are few retrospective reports covering this subject, and even fewer enrolled patients. However, researchers agree that hepatic resection for SCLM is a viable treatment modality in multidisciplinary treatment.

Based on previous studies, some variables (the number of hepatic metastases, the time interval of DFS, and $<3$ positive lymph nodes etc.) have been suggested as potential prognostic factors of hepatic resection after SCLM [1-4]. In our study, we could not analyze the prognostic factors owing to the small number of enrolled patients. We considered hepatic resection in a select few SCLM patients. Despite strict patient selection (solitary lesion and absence of extrahepatic lesion), by using preoperative imaging techniques, it is difficult to diagnose extrahepatic metastases and the number of hepatic metastases accurately. In spite of this, diagnostic laparoscopic evaluation is still recommended, as it is difficult to get accurate information due to adhesions after gastrectomy. We did not perform laparoscopic evaluation, and one case changed in management. Preoperative diagnostic accuracy was only 57.1\% (4/7). Three cases had multiple hepatic metastases and they had poor prognoses. In a recent study, survival benefit was present in synchronous hepatic metastasis when R0 resection was possible [6]. Although our data has limitations, the time interval of DFS might not be an important prognostic factor for the hepatic resection of SCLM. HER2 is also a prognostic factor in stomach cancer [7-9]. In our study, two patients with HER2-negative tumors achieved long-term survival and one patient with an HER2-positive tumor, who was treated with trastuzumab plus systemic chemotherapy, had stable disease status.

This study has some limitations. First, this was a retrospective 
study. Second, all patients with single and unilateral metastasis underwent hepatic resection and we could not find any resection cases with single and unilateral metastasis. Third, owing to the small numbers of patients, no statistical comparisons could be made. Although this study included a small number of patients and has limitations, our data suggests that liver resection in patients with SCLM, with single metastases and HER2-negative tumors, can result in an improved long-term survival rate. Therefore, liver resection can be considered as an effective treatment modality in multidisciplinary treatment of SCLM patients.

\section{CONFLICT OF INTEREST}

No potential conflict of interest relevant to this article was reported.

\section{REFERENCES}

1. Dittmar Y, Altendorf-Hofmann A, Rauchfuss F, Gotz M, Scheuerlein $\mathrm{H}$, Jandt $\mathrm{K}$, et al. Resection of liver metastases is beneficial in patients with gastric cancer: report on 15 cases and review of literature. Gastric Cancer 2012;15:131-6.

2. Tiberio GA, Ministrini S, Gardini A, Marrelli D, Marchet A, Cipollari $\mathrm{C}$, et al. Factors influencing survival after hepatectomy for metastases from gastric cancer. Eur J Surg Oncol 2016;42:1229-35.

3. Petrelli F, Coinu A, Cabiddu M, Ghilardi M, Borgonovo K, Lonati
$\mathrm{V}$, et al. Hepatic resection for gastric cancer liver metastases: a systematic review and meta-analysis. J Surg Oncol 2015;111:1021-7.

4. Oguro S, Imamura H, Yoshimoto J, Ishizaki Y, Kawasaki S. Liver metastases from gastric cancer represent systemic disease in comparison with those from colorectal cancer. J Hepatobiliary Pancreat Sci 2016;23:324-32.

5. Markar SR, Mikhail S, Malietzis G, Athanasiou T, Mariette C, Sasako $\mathrm{M}$, et al. Influence of surgical resection of hepatic metastases from gastric adenocarcinoma on long-term survival: systematic review and pooled analysis. Ann Surg 2016;263:1092-101.

6. Tatsubayashi T, Tanizawa Y, Miki Y, Tokunaga M, Bando E, Kawamura T, et al. Treatment outcomes of hepatectomy for liver metastases of gastric cancer diagnosed using contrast-enhanced magnetic resonance imaging. Gastric Cancer 2017;20:387-93.

7. Gomez-Martin C, Garralda E, Echarri MJ, Ballesteros A, Arcediano A, Rodriguez-Peralto JL, et al. HER2/neu testing for anti-HER2based therapies in patients with unresectable and/or metastatic gastric cancer. J Clin Pathol 2012;65:751-7.

8. Janjigian YY, Werner D, Pauligk C, Steinmetz K, Kelsen DP, Jager E, et al. Prognosis of metastatic gastric and gastroesophageal junction cancer by HER2 status: a European and USA international collaborative analysis. Ann Oncol 2012;23:2656-62.

9. Boku N. HER2-positive gastric cancer. Gastric Cancer 2014;17:112. 